\title{
Fabrication of Conductometric Gas-Sensing Films by Selected Area Chemical-Vapor Deposition
}

\author{
Sanjeev Majoo and Johannes W. Schwank \\ Dept. of Chemical Engineering, University of Michigan, Ann Arbor, MI 48109 \\ John L. Gland \\ Depts. of Chemistry and Chemical Engineering, University of Michigan, Ann Arbor, MI 48109
}

\begin{abstract}
A new selected-area chemical-vapor deposition (CVD) process to fabricate thin sensing films on a micromachined silicon-based, conductometric gas-sensor structure is reported. The CVD method is self-lithographic and does not require the use of masks to define the thin-film region. Since the films are deposited after silicon processing, the selection of film materials is not constrained by their compatibility with the etchants used in silicon micromachining. The presence of conductance electrodes underneath the growing film permits in-situ monitoring of film resistance. The rapid thermal response of the device allows precise termination of film growth once the film resistance has reached a value suitable for gas sensing. The application of this new CVD method is demonstrated for growth of thin platinum films, which are used for sensing of $\mathrm{O}_{2}$ and $\mathrm{H}_{2}$ gases.
\end{abstract}

\section{Introduction}

There is a growing demand for reliable, sensitive, selective, and inexpensive gas sensors. Advances in silicon micromachining combined with lithographic patterning technology widely used in modern microelectronics have made the miniaturization of sensors possible. These microsensors offer several advantages over conventional analytical instruments. They have lower fabrication costs and lower power consumption, and they can easily be deployed in a distributed environment for on-line or remote sensing.

A recently developed conductometric gas-sensing device contains a discontinuous $\mathrm{Pt} / \mathrm{Ti}$ thin film that undergoes changes in resistance upon adsorption or surface reaction with gas molecules of interest (Johnson et al., 1988, 1990, 1994; Najafi et al., 1994; DiBattista et al., 1995; Patel et al., 1996, 1997). A mechanistic study of gas-sensing characteristics of ultrathin $\mathrm{Pt} / \mathrm{TiO}_{x}$ films has shown that the film-resistance changes upon exposure to hydrogen are most likely due to the formation of surface hydroxyl groups, titanium hydride bonds, and the removal of lattice oxygen, leading to vacancy defects (Walton, 1997; Walton et al., 1997). As film thickness decreases, conductance becomes increasingly dominated by charge-carrier concentration and mobility on the surface and in subsurface layers. Due to the high surface-to-volume ratio

Correspondence concerning this article should be addressed to J. W. Schwank of such thin films, their conductance can be strongly influenced by surface adsorption and surface reaction. For discontinuous films, thermionic emission and activated tunneling have been proposed as possible mechanisms of conduction (Hartman, 1963). Adsorbed surface species can modify the net film conductance by altering charge-carrier mobility and charge-carrier concentration (Murgulescu and Ionescu, 1971).

For practical gas-sensing applications, one of the major challenges is to differentiate between different types of gas molecules. The analysis of complex gas mixtures represents a multivariable problem that cannot be solved based on data input from a single front-end sensing element. Silicon micromachining opens up an opportunity to fabricate sensors with low power consumption, a key issue in portable, batteryoperated sensors. The conductometric gas-sensing device used in this work contains an integrated microheater underneath a selectively micromachined dielectric membrane. This membrane, which has a low thermal mass and excellent thermal isolation to optimize the thermal response, is micromachined from the wafer bulk, using an ethylenediamine pyrazine pyrocatechol (EDP) etch. However, many desirable and chemically selective thin-film materials are completely etched along with silicon during this micromachining process.

Furthermore, silicon technology is attractive for realization of sensor arrays that contain different film materials (John- 
son et al., 1990; Najafi, 1992; Cavicchi et al., 1993; Semancik et al., 1995) to provide a characteristic fingerprint response to gas mixtures. It is desirable to custom deposit a variety of films on prefabricated sensor structures, without exposing the film to the etch chemicals. In this article, maskless, thermally activated chemical-vapor deposition (CVD) of Pt films on a selected area of this prefabricated gas-sensor structure is described. The film growth is continuously monitored by conductance electrodes. Film deposition is terminated at film resistance levels suitable for sensing of oxygen and hydrogen gases. A feasibility study of film deposition on a two-element discrete sensor array is also presented.

\section{Experimental Procedures}

To overcome the limitation in the selection of chemicalsensing films that would be incompatible with the EDP etch, a variation of the original device structure (Najafi et al., 1994) and fabrication method has been developed.

\section{Sensor fabrication and structure}

Figure 1 shows a top view and cross-sectional view of the modified device structure. The sensor fabrication process begins with a solid-source boron diffusion into a patterned 550 $\mu \mathrm{m}$ Si wafer to define the rim area of the silicon chip from the front, and the pattern for defining the final membrane and die size from the back. A thermal oxide is then grown,

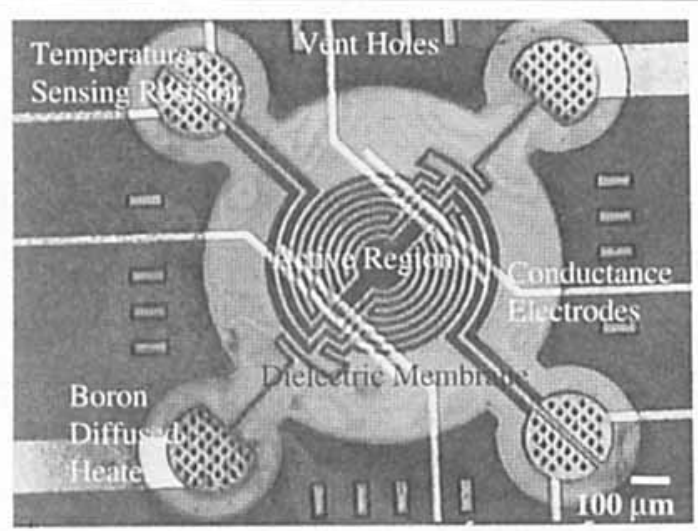

(a)

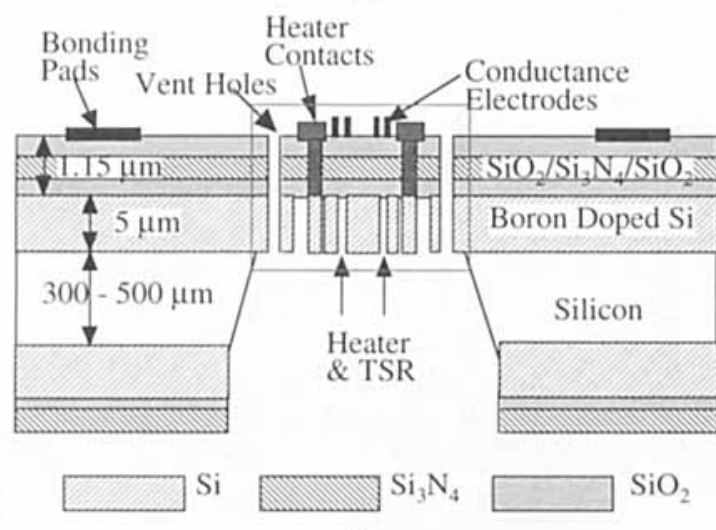

(b)

Figure 1. Gas sensor structure in (a) top view and (b) cross-sectional view. followed by boron doping and patterning for the temperature-sensing resistors (TSRs) and a meandering heater on the back. A three-layered sandwich structure containing films of LPCVD $\mathrm{SiO}_{2}, \mathrm{Si}_{3} \mathrm{~N}_{4}$, and $\mathrm{SiO}_{2}$ is deposited on the front side. The oxide layers are under compressive stress, whereas the nitride layer is under tensile stress. In order to provide a stress-relieved membrane over the typical operating temperature range of the sensor, the thicknesses of these three layers are adjusted. For example, for sensors expected to operate at a higher temperature, $250-\mathrm{nm}$ bottom $\mathrm{SiO}_{2}, 500-\mathrm{nm} \mathrm{Si}{ }_{3} \mathrm{~N}_{4}$, and $400-\mathrm{nm}$ top $\mathrm{SiO}_{2}$ thicknesses are used. A buffered HF etch and a plasma etch are used to open contacts through the $\mathrm{SiO}_{2}$ and $\mathrm{Si}_{3} \mathrm{~N}_{4}$ to the buried heater and the TSRs. Strips of $20-\mathrm{nm}$ Ti under $150-\mathrm{nm} \mathrm{Pt}$ are sputtered and lifted off to define the four-point conductance electrodes. Additional metal layers of $\mathrm{Ti}$ and $\mathrm{Au}$ are placed in the external pad areas to facilitate Au wire bonding. The bulk silicon underneath the three-layered dielectric stack is etched with EDP to create the dielectric membrane with integrated heater and TSRs. Finally, the active sensing film is deposited after microfabrication, using a novel selected area CVD (SACVD) process (Gland et al., 1994; Majoo, 1997; Majoo et al., 1994, 1995).

\section{Film-deposition process}

Film deposition is carried out at low pressures in a cold-wall ultrahigh-vacuum (UHV) reactor with a typical base pressure of $10^{-8}$ torr. The device is mounted on a transistor outline (TO) header and a 10-pin electrical feedthrough (see Figure 2). The feedthrough is introduced directly into the chamber and externally connected to a data-acquisition and temperature-control system. This allows accurate and rapid temperature programmability of a well-defined circular region in the center of the membrane, $500 \mu \mathrm{m}$ in diameter, hereafter referred to as the "active region" of the device. The insulating dielectric membrane and the thick silicon rim surrounding the active region provide thermal isolation.

For deposition of platinum films, tetrakis trifluorophosphine platinum, $\operatorname{Pt}\left(\mathrm{PF}_{3}\right)_{4}$, is used (Rand, 1973). The precursor compound is purified by freeze-pump-thaw cycles and the

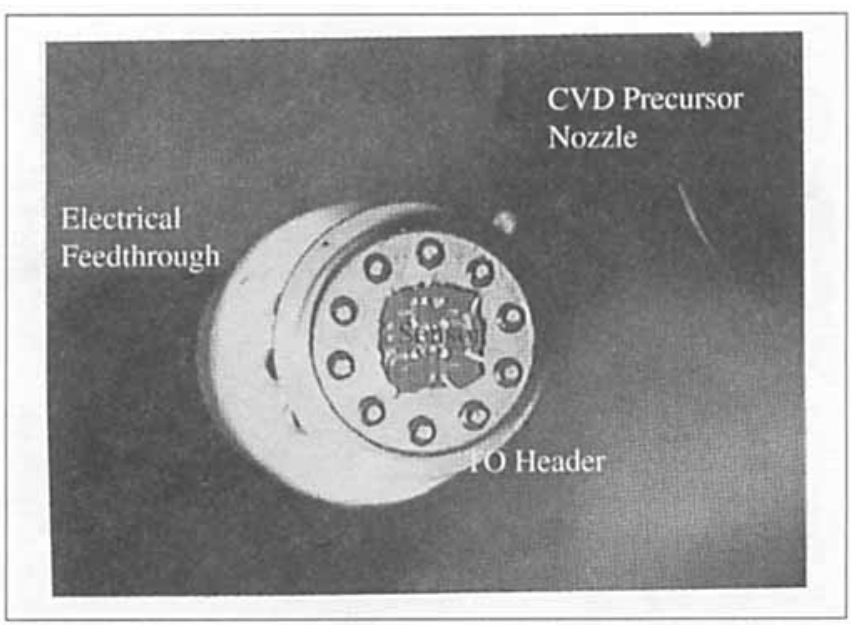

Figure 2. Sensor mounted on a To header and an electrical feedthrough, and inserted into the UHV CVD reactor. 
vapors are directly dosed into the chamber. While the bulk gas phase in the CVD reactor remains at room temperature, the active region is resistively heated, by driving a current through the boron-doped meandering heater, to a temperature above the threshold for thermal decomposition of the precursor. Only molecules in direct thermal contact with the heated, active region undergo pyrolysis. In this manner, film growth is confined exclusively to the active region of the sensor (see Figure 3). Although the conductance electrodes are protruding $170 \mathrm{~nm}$ above the planar dielectric membrane, the localized thermal CVD method tends to give good step coverage of thin sensing films. Two modes of thin-film processing are used: isothermal and pulsed.

In the isothermal mode of operation, the temperature of the active region is first ramped up in vacuum to a desired CVD temperature and held at this level. Then, precursor vapors are introduced through a leak valve into the CVD reactor until a desired constant reactor pressure is reached. Once an appropriate film resistance is obtained, the heater current is switched off, rapidly decreasing the temperature of the active region to room temperature and terminating the deposition process. Finally, the precursor vapors are pumped out and the reactor pressure is brought back to its base value. In the pulsed mode, a constant precursor pressure is first established in the CVD reactor. Then, the active-region temperature is switched from ambient to the CVD temperature regime, held at the CVD temperature for a few seconds, and then immediately brought back to the ambient temperature. These temperature pulses are repeated until the film resistance reaches a suitable value. Finally, the precursor vapors are pumped out of the reactor with the active region of the device at ambient temperature. The key parameters affecting the pulsed deposition process are the magnitude and duration of each temperature pulse.

The feasibility of using this SACVD process for deposition of sensing films on an array structure has been analyzed. Two discrete sensor structures are mounted in the CVD reactor next to each other. The platinum precursor vapors are introduced to establish a reactor pressure of $1 \times 10^{-2}$ torr. One device is kept at ambient temperature, while the active re-

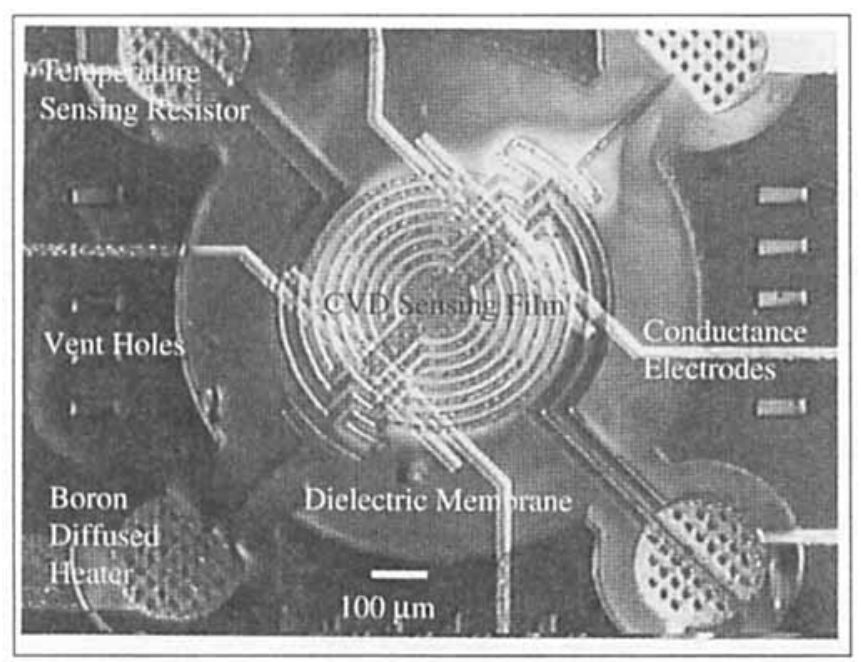

Figure 3. Sensor with a self-lithographic Pt CVD film confined to the active region.

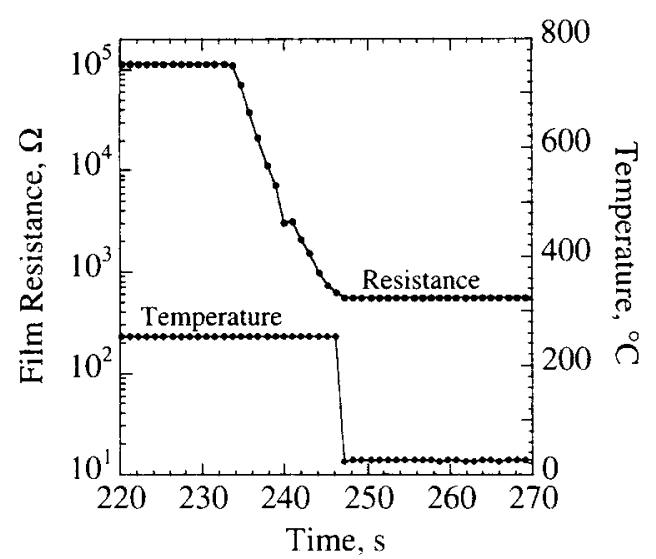

Figure 4. Pt CVD at a constant temperature of $250^{\circ} \mathrm{C}$ and a constant pressure of $5 \times 10^{-4}$ torr.

gion of the other device is heated to $200^{\circ} \mathrm{C}$. The platinum-film deposition is confined only to the active region of the heated device.

\section{Results and Discussion}

Figures 4 and 5 show a typical plot of resistance and temperature vs. time during Pt-film growth for two different cases: isothermal processing and pulsed deposition. The initial resistance as measured by four-point electrodes is greater than the upper limit of our data-acquisition system $\left(\sim 10^{5} \Omega\right)$. This is expected, since the electrodes are in contact with an insulating dielectric membrane.

For isothermal processing at $250^{\circ} \mathrm{C}$ and $5 \times 10^{-4}$ torr (see Figure 4), it takes about $234 \mathrm{~s}$ until the resistance values become low enough to be tracked by the data-acquisition system. The resistance decreases until the membrane temperature is lowered to room temperature $\left(25^{\circ} \mathrm{C}\right)$. From then on, the film resistance remains constant at $540 \Omega$.

Figure 5 shows a pulsed deposition carried out at $2 \times 10^{-5}$ torr with temperature pulses of varying duration between ambient and $180^{\circ} \mathrm{C}$. With each temperature pulse, the film resistance shows a sharp decrease. At the end of each temperature pulse, the resistance does increase, but it remains at a value lower than the room-temperature resistance prior to

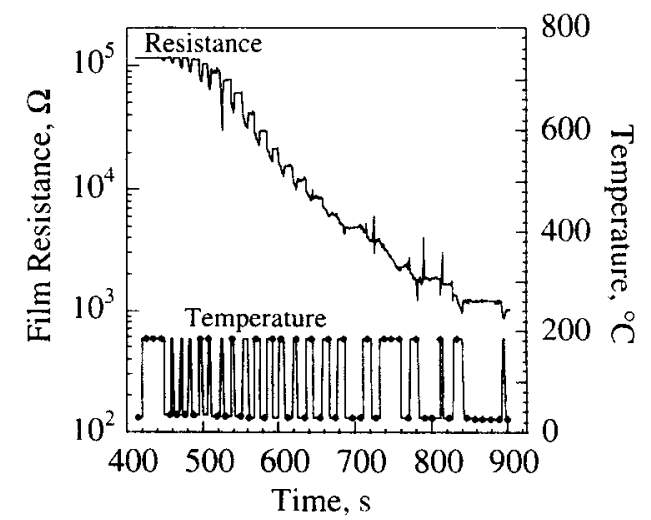

Figure 5. Pt CVD at a constant pressure of $2 \times 10^{-5}$ torr and temperature pulses between room temperature and $180^{\circ} \mathrm{C}$. 
the temperature pulse. Overall, the room-temperature resistance shows a continuous downward trend as temperature pulses are repeated. As expected, temperature pulses of longer duration lead to more pronounced decreases in film resistance. This experiment demonstrates the flexibility of the temperature control system permitting well-controlled, stepwise film growth until a desired film resistance is reached.

The SACVD method is also useful for modification of conventionally deposited sensing films. Figure 6 shows how an evaporated $\mathrm{Pt} / \mathrm{Ti}$ film, having a very high resistance $(25 \mathrm{k} \Omega$ ) unsuitable for microsensing, can be modified to lower the resistance. It is important to note that the SACVD Pt film is added on top of the electrodes, while the original, evaporated $\mathrm{Pt} / \mathrm{Ti}$ film is underneath the electrodes. The presence of the Pt/Ti film appears to catalyze the Pt CVD. In the first $50 \mathrm{~s}$, the resistance decreases in a stepwise fashion (see insert in Figure 6), followed by a slow decrease in resistance, and ending in a sharp drop in resistance to a final value of $10 \Omega$. At this point, the heater current is switched off and the temperature is lowered to room temperature to terminate film growth. An atomic-force micrograph (AFM) of the resulting film (Figure 7) indicates columnar growth of Pt structures up to $400 \mathrm{~nm}$ in height. We attribute the sharp drop in resistance after $600 \mathrm{~s}$ to coalescence of these Pt columns. In contrast, $\mathrm{Pt}$ CVD films deposited directly on $\mathrm{SiO}_{2}$ (the top layer of the dielectric membrane) tend to consist of discontinuous Pt islands less than $100 \mathrm{~nm}$ in diameter (Figure 8).

Figure 9 illustrates a typical response of a $\mathrm{Pt}$ CVD film deposited directly on $\mathrm{SiO}_{2}$ at low partial pressures of gases. With the active region at a temperature of $400^{\circ} \mathrm{C}$, the resistance increases slightly on exposure to oxygen and then returns toward the base line when oxygen is evacuated. However, upon exposure to hydrogen, the resistance decreases immediately by more than $10 \Omega$ and then returns toward the base line once the hydrogen is evacuated. These initial results show the sensitivity and promising selectivity of Pt CVD films. Acceptor gases such as oxygen inject holes into the conduction band of metals, resulting in an increase in resistance due to a decrease in charge-carrier concentration. Donor gases, such as hydrogen, inject electrons into the conduction band, leading to an increase in the concentration of charge carriers and a decrease in resistance. Further research will be required to develop a mechanistic model for the se-

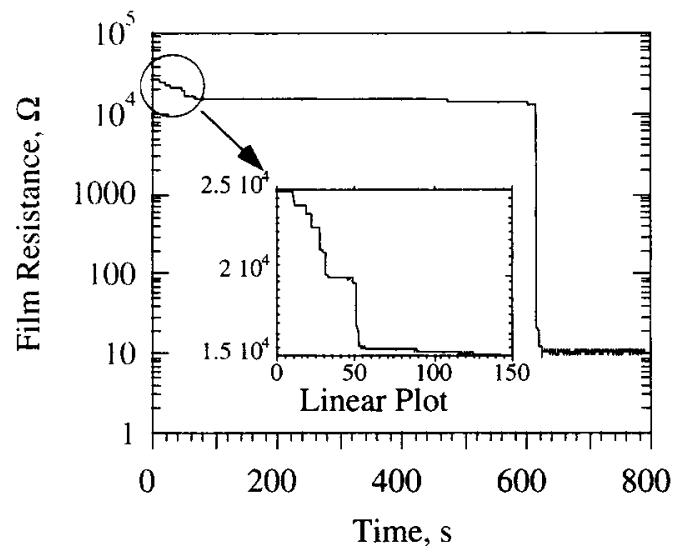

Figure 6. Postdeposition modification of an existing, evaporated $\mathrm{Pt} / \mathrm{Ti}$ film.

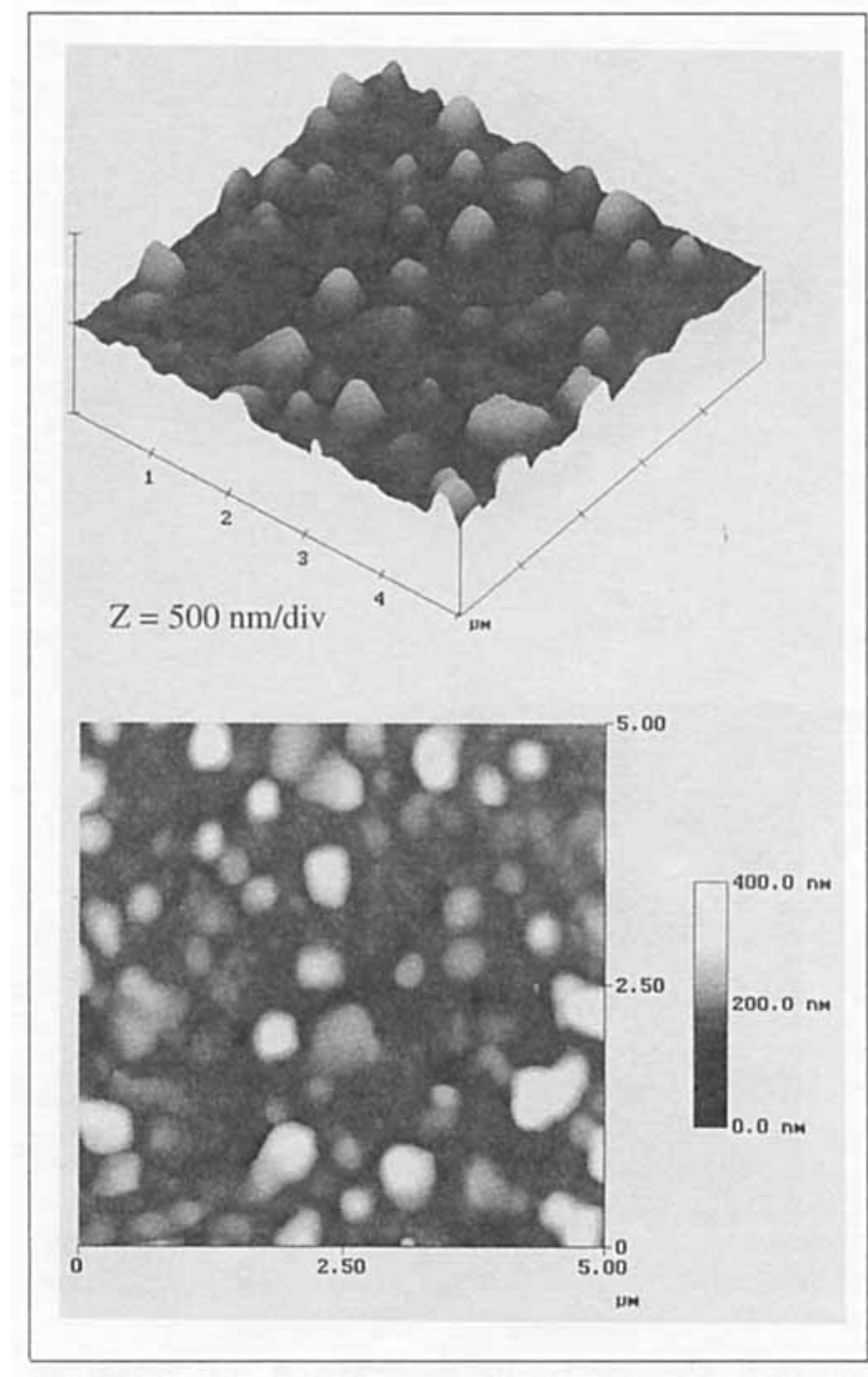

Figure 7. Atomic-force micrograph of a Pt CVD film deposited on top of an existing evaporated $\mathrm{Pt} / \mathrm{Ti}$ film.

lected-area CVD process and to fully understand the response of these platinum sensing films to a variety of gases.

\section{Conclusions}

We have demonstrated the feasibility of using heated dielectric membrane structures to initiate maskless, thermally activated CVD of Pt films on a geometrically well-defined area, $500 \mu \mathrm{m}$ in diameter. A four-point electrode configuration located underneath the growing film is used to continuously monitor the film growth by in situ resistance measurements. The fast thermal response of the dielectric membrane permits isothermal or temperature-pulsed deposition, and termination of film growth once a desired resistance value is reached. The SACVD method is used to deposit self-lithographic sensing films on the active region of sensors after the etching process. It is also used to modify existing $\mathrm{Pt} / \mathrm{Ti}$ films to optimize their film resistance for sensing applications. The platinum CVD films are used to detect oxygen and hydrogen gases at low partial pressures. The SACVD process described here lays the ground for efficient fabrication of thin-filmbased sensor arrays. The preliminary results obtained with a 


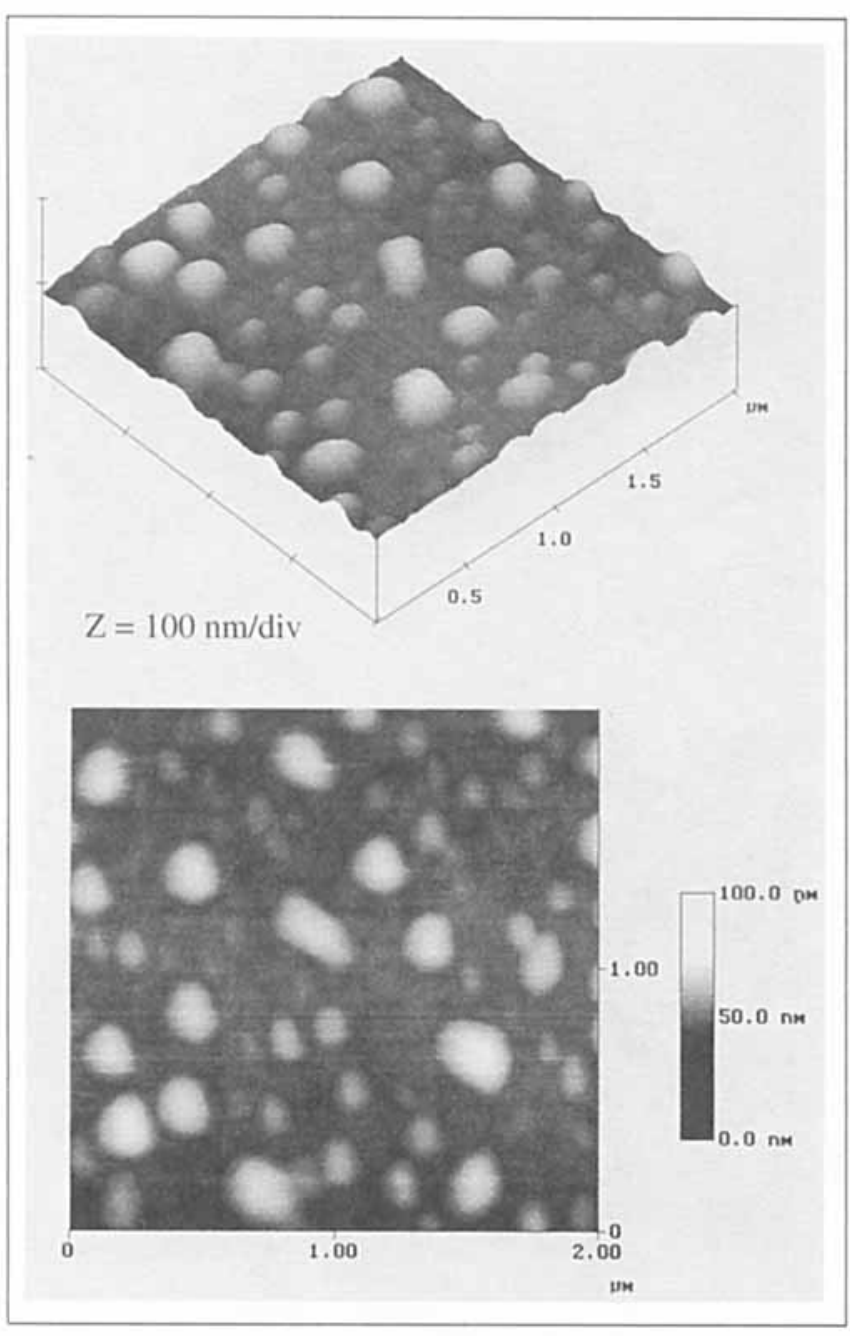

\section{Figure 8. Atomic-force micrograph of a Pt CVD film de- posited directly on the dielectric membrane.}

set of two discrete sensor structures mounted side by side in the CVD reactor show that this method is promising for custom deposition of different films on active regions of discrete or monolithically integrated arrays.

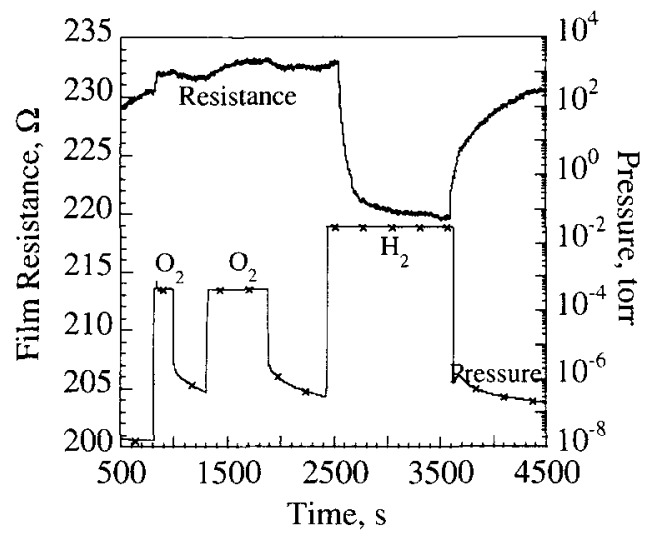

Figure 9. Response of a Pt CVD film with the active region at $400^{\circ} \mathrm{C}$ to low-pressure pulses of hydrogen and oxygen.

\section{Acknowledgments}

Financial support for part of this work provided by DARPA under Contract DABT63-C-0111 is gratefully acknowledged. The authors also acknowledge Prof. Kensall D. Wise for his contribution to integrated sensor technology, Mr. Michael DiBattista for atomic force micrographs, and Drs. Paul L. Bergstrom and Sanjay V. Patel for the development of automated data-acquisition and control system.

\section{Literature Cited}

Cavicchi, R. E., G. E. Poirier, S. Semancik, J. S. Suehle, and M. Gaitan, "Development of CMOS-Based Gas Microsensor Arrays with Materials and Kinetic Selectivity," Proc. AVS Symp., Orlando, FL, p. 290 (1993).

DiBattista, M., S. V. Patel, K. D. Wise, J. L. Gland, J. Mansfield, and J. W. Schwank, "Characterization of Multilayer Thin Film Structures for Gas Sensor Applications," Proc. Mater. Res. Soc. Symp., MRS Spring Meeting, San Francisco, CA, Apr. 17-21, p. 477 (1995)

Gland, J. L., J. W. Schwank, and K. D. Wise, "In Situ Monitoring and Growth of Thin Films by Means of Selected Area CVD,"U.S. Patent No. 5,296,255 (1994).

Hartman, T. E., "Electrical Conduction in Discontinuous Thin Metal Films," J. Appl. Phys., 34(4), 943 (1963).

Johnson, C. L., K. D. Wise, and J. W. Schwank, "A Thin-Film Gas Detector for Semiconductor Process Gases," IEEE Dig. Tech. Pap., 662 (1988).

Johnson, C. L., J. W. Schwank, and K. D. Wise, "Ultra Thin-Film Gas Detector," U.S. Patent No. 4,953,387 (1990).

Johnson, C. L., J. W. Schwank, and K. D. Wise, "Integrated UltraThin-Film Gas Sensors," Sens. Actuators B, 20, 55 (1994).

Majoo, S., "Selected-Area Chemical Vapor Deposition of Thin Films for Conductometric Microelectronic Chemical Sensors," PhD Diss., Univ. of Michigan, Ann Arbor (1997).

Majoo, S., J. W. Schwank, J. L. Gland, and K. D. Wise, "In Situ Monitoring of Pt Thin Film Growth Using CVD on Micro Hot Plates," Preprints Topical Conf. on The Synthesis and Processing of Electronic Materials, AIChE Meeting, San Francisco, CA, p. 242 (1994).

Majoo, S., J. W. Schwank, J. L. Gland, and K. D. Wise, "A Selected-Area CVD Method for Deposition of Sensing Films on Monolithically Integrated Gas Detectors," IEEE Electron Device Lett., 16(6), 217 (1995).

Murgulescu, I. G., and N. I. Ionescu, "The Effect of Oxygen Chemisorption on the Electrical Properties of Thin Copper and Silver Films," Thin Solid Films, 7, 355 (1971).

Najafi, N., "A Generic Smart Sensing System Utilizing a Multi-Element Gas Analyzer," PhD Diss., Univ. of Michigan, Ann Arbor (1992).

Najafi, N., K. D. Wise, and J. W. Schwank, "A Micromachined Ultra-Thin-Film Gas Detector," IEEE Trans. Electron Devices, ED41(10), 1770 (1994).

Patel, S. V., M. DiBattista, J. L. Gland, and J. W. Schwank, "Survivability of a Silicon-Based Microelectronic Gas Detector Structure for High Temperature Flow Applications," Sens. Actuators B, 37, 27 (1996)

Patel, S. V., M. DiBattista, J. L. Gland, and W. Schwank, "A Microelectronic Hydrogen Gas Sensor," Amer. Lab., 29, 18N (1997).

Rand, M. J., "Chemical Vapor Deposition of Thin-Film Platinum," $J$. Electrochem. Soc., 120, 686 (1973).

Semancik, S., R. E. Cavicchi, K. G. Kreider, J. S. Suehle, and P. Chaparala, "Selected-Area Deposition of Multiple Active Films for Conductometric Microsensor Arrays," Proc. Transducers, Int. Conf. on Solid-State Sensors and Actuators, Stockholm, p. 831 (1995).

Walton, R. M., "Mechanistic Studies of Platinum-Titania and Platinum-Alumina Thin Films for Microchemical Gas Sensors," PhD Diss., Univ. of Michigan, Ann Arbor (1997).

Walton, R. M., J. W. Schwank, and J. L. Gland, "Gas Sensing Characteristics of Ultra Thin $\mathrm{TiO}_{2-x}$ Films Investigated with XPS, TPD and In Situ Resistance Measurements," Surf. Interf. Anal., 25, 76 (1997).

Manuscript received Oct. 28, 1996, and revision received June 23, 1997. 